\title{
Desintoxicación ambulatoria de alcohol: una opción segura
}

\author{
JACINTO MOSQUeRA NOGUEIRA*; CARMEN GUIMERANS FREIJEIRO**; BERTA GÓMEZ BEN**; \\ NURIA DAVILA DOMÍNGUEZ*** \\ *Médico. \\ **Psicóloga Clínica. \\ ***Trabajadora Social
}

Enviar correspondencia a:

Jacinto Mosquera. Unidad asistencial de tratamiento de alcoholismo "ASVIDAL" C/ Escultor Gregorio Fernández, nº 8, bajo. 36204 Vigo (Pontevedra). E-mail: info@alcoholasvidal.com

\section{RESUMEN}

OBJETIVOS: demostrar la seguridad del proceso de desintoxicación ambulatoria de la dependencia alcohólica.

METODOLOGÍA: análisis retrospectivo de 100 desintoxicaciones indicadas en la Unidad de Alcoholismo de Vigo, recogiendo las siguientes variables: sexo, edad, ámbito de desintoxicación, fármaco utilizado para la desintoxicación, otros fármacos para deshabituación (aversivos, anti-craving), desintoxicación completada, sintomatología de abstinencia leve, moderada o grave. Se utilizó para la desintoxicación Clometiazol en 66 casos, Tetrabamato en 29 y benzodiacepinas en 3 casos. Se cruzaron las variables estudiadas con la presencia o no de síntomas de abstinencia, utilizando la prueba del chi cuadrado.

RESULTADOS: Se completó la desintoxicación ambulatoria en 88 casos sin la aparición de síntomas; aparecieron síntomas leves o moderados en 15 casos y ninguno presentó síntomas graves de abstinencia. Solo 2 casos precisaron ingreso hospitalario. Ninguna variable se correlaciona con la aparición de síntomas de abstinencia.

CONCLUSIÓN: La desintoxicación ambulatoria es un proceso seguro, una vez excluida causa de ingreso.

Palabras clave: desintoxicación, ambulatoria, alcohol, dependencia, seguridad.

\section{ABSTRACT}

GOAL: to show that out-patient detoxification is a safe process to treat alcoholic addiction.

METHODS: a retrospective analysis of 100 detoxifications reported in the Alcoholism Unit of Vigo was performed. The following variables were included in the study: gender, age, detoxification environment, main drug used for detoxification, other drugs used for alcoholism treatment (aversive, anticraving), complete detoxification; symptoms of slight, moderate or serious abstinence. The following drugs were used for detoxification: Clometiazol in 66 cases, Tetrabamato in 29 and benzodiazepines in 3. A comparison analysis of these variables was carried out using the chi square test.

RESULTS: The out-patient detoxification was successfully completed in 88 cases; slight or moderate symptoms of abstinence showed up in 15 cases, and none of them had serious symptoms of abstinence. Only 2 cases needed to be hospitalized.

CONCLUSION: Out-patient detoxification is a safe process, if a reason for hospitalization is excluded.

Key words: detoxification, ambulatory, alcohol, dependency, safety.

en fases evolucionadas de la enfermedad alcohólica. Por ello nunca se insistirá lo bastante en el papel clave de la Atención Primaria de Salud, en las fases primaria, secundaria y terciaria de la prevención.

Cuando se diagnostica una dependencia alcohólica, se debe remitir al sujeto al dispositivo correspondiente del Área Sanitaria': Unidad de Alcoholismo, Unidad de Conductas Adictivas, Unidad de Drogodependencias, Unidad de Salud Mental... La gran complejidad 
de la patología y de las diferentes intervenciones a realizar, justifican la derivación a un dispositivo multidisciplinar que atienda las múltiples complicaciones de la enfermedad.

Pero la Atención Primaria de Salud debe seguir implicada en el proceso, en todas las fases y, singularmente, en la de desintoxicación. La posición privilegiada del médico y enfermero de Atención Primaria permite una continuada relación motivacional y un seguimiento de los distintos aspectos que la enfermedad genera.

En este abordaje se definen clásicamente tres fases: desintoxicación, deshabituación y reinserción. Pero no son unas fases "estanco" sino que se pueden, y, generalmente se deben, solapar, de tal forma que al mismo tiempo que se desintoxica al paciente, a veces es necesaria una intervención de emergencia social (como por ejemplo, conseguirle un sitio donde dormir).

La desintoxicación es el proceso de tratamiento del síndrome de abstinencia provocado por el cese o reducción del consumo de alcohol.

Este proceso de desintoxicación, se puede realizar de forma ambulatoria o en régimen de ingreso hospitalario en sala de psiquiatría. Decidir una u otra es una de las primeras cuestiones tras realizar una historia alcohológica ${ }^{2,3}$.

En la Unidad de Alcohología de Vigo (ASVIDAL) utilizamos como indicaciones de ingreso las siguientes:

1. Falta de persona que acompañe al paciente en su desintoxicación.

2. Patología psiquiátrica concomitante que precise ingreso por sí misma.

3. Riesgo familiar o social.

4. Aparición de síntomas de abstinencia graves a pesar de tratamiento oral a dosis plenas.

Planteadas así las cosas, nos interrogamos sobre la seguridad del tratamiento ambulatorio, consistente en evitar farmacológicamente y con medidas de sostén el síndrome de abstinencia. Los sedantes más usados en nuestro medio son el Clometiazol, el Tetrabamato (hasta su retirada del mercado) el Tiaprizal, las Benzodiacepinas 4, 5, 6, 7, 8, 9. En las desintoxicaciones ambulatorias los fármacos se dosifican de forma fija y descendente ${ }^{10}$.

En algunas ocasiones se inicia de forma simultánea la toma de fármacos más específicos para la deshabituación ${ }^{11,12}$ como los aversivos, la naltrexona o el acamprosato.

Nos propusimos comprobar, mediante la presente investigación, la seguridad del proceso de desintoxicación ambulatoria.

\section{MATERIAL Y MÉTODOS}

Realizamos una evaluación retrospectiva de las 100 últimas desintoxicaciones realizadas en el año 1999 en los pacientes que acuden a tratamiento en la Unidad de Alcohología de Vigo (ASVIDAL), de referencia para el tratamiento de esta patología en el área sanitaria de Vigo (Pontevedra Sur)

De las historias clínicas extrajimos los siguientes datos: sexo, edad y ámbito de desintoxicación.

En los casos de desintoxicación ambulatoria, se siguieron extrayendo datos como:

- Fármaco utilizado en la DTX

- Otros fármacos utilizados como: aversivo, naltrexona, acamprosato.

- ¿Se completó el tratamiento?

- ¿Apareció sintomatología de abstinencia? ¿Este fue leve o grave?

\section{RESULTADOS}

De las 100 historias revisadas, el 75\% correspondían a varones y el $25 \%$ a mujeres (Figura $\boldsymbol{n}^{\circ}$ 1), lo cual se corresponde con la proporción habitual de los pacientes de la Unidad; esta proporción de mujeres es algo mayor que en otras series, en que el sexo femenino no suele representar más del $20 \%$.

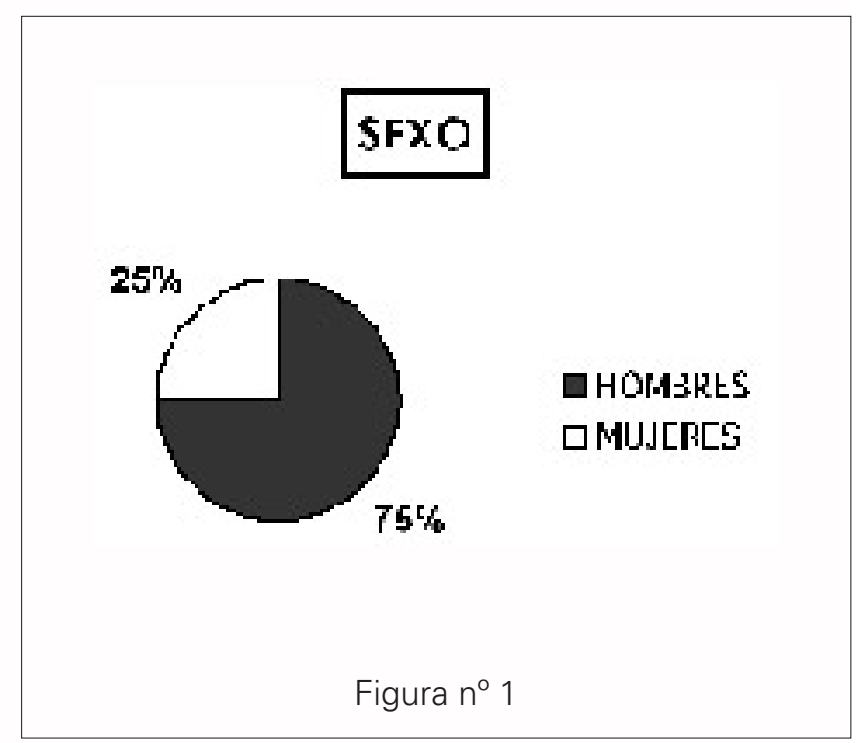

La edad media de los pacientes fue del 44,08 con un rango de edad de 21 a 71 años. También esta media de edad es la habitual en la Unidad. 


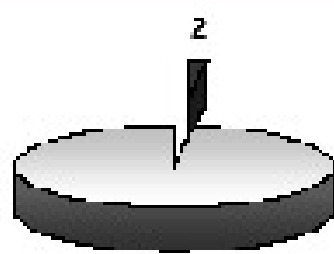

97

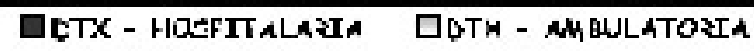

Figura $\mathrm{n}^{\circ} 2$

Solo en 2 casos se remitió al paciente para desintoxicación hospitalaria. (Figura $\boldsymbol{n}^{\mathbf{0}}$ 2) En uno de los casos se trataba de una dependencia alcohólica con un cuadro de delirio celotípico con grave riesgo familiar y en el otro caso, la indicación de ingreso fue la falta de persona acompañante responsable en un paciente con una enfermedad alcohólica muy evolucionada.

En los 98 casos restantes se siguió un proceso ambulatorio. El fármaco utilizado fue (Figura $\boldsymbol{n}^{\circ} \mathbf{3}$ ):

- "Clometiazol": 66 casos

- "Tetrabamato": 29 casos

- "Benzodiazepina": 3 casos

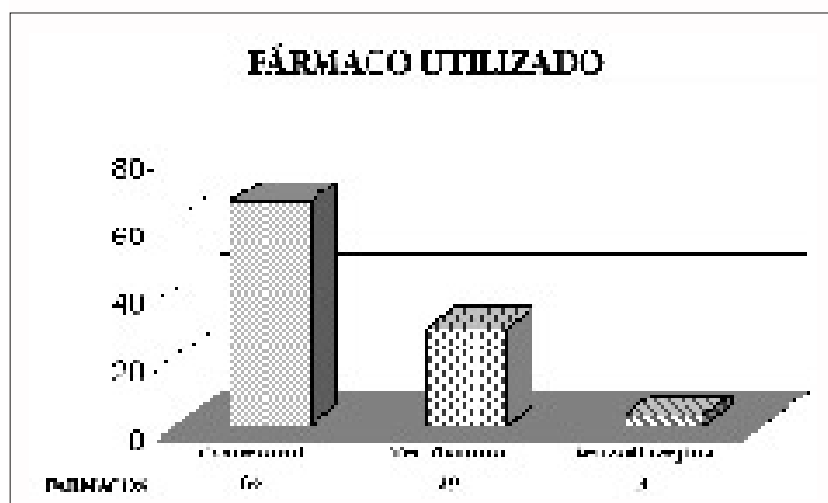

Figura $n^{\circ} 3$

En 38 pacientes se asoció un disuasorio (disulfiram ó cianamida cálcica) en algún momento de la fase de desintoxicación cuando se encontraron dificultades para conseguir abstinencia completa; en ningún caso, fue prescrito al inicio del proceso.

La adicción de naltrexona o acamprosato se indicó cuanto el paciente refirió intenso deseo de consumo (craving) tras iniciar la desintoxicación. En 6 casos se asoció naltrexona y en 3 acamprosato.

El proceso de desintoxicación se completó satisfactoriamente en 88 casos. Los 10 restantes abandonaron el tratamiento. (Figura $\boldsymbol{n}^{\circ} \mathbf{4}$ )

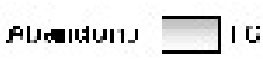

Enmplats

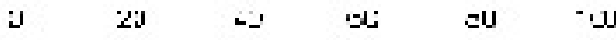

口Completo ப $\lrcorner$ Abandono

Figura $n^{\circ} 4$

A pesar del tratamiento, aparecieron síntomas leves de abstinencia en 15 casos y, lo que es más importante, no aparecieron síntomas graves en ningún caso.

Para completar el análisis de los datos, cruzamos las distintas variables estudiadas con el hecho de haber o no completado el tratamiento y con el hecho de haber o no presentado sintomatología de abstinencia. A las diferencias encontradas, le pasamos el test del Chi-cuadrado: en ningún caso se alcanzó la significación estadística.

\section{DISCUSIÓN}

El hecho de que no hayan aparecido síntomas graves en ninguno de los casos estudiados, corrobora nuestra impresión previa de que una adecuada desintoxicación ambulatoria es un proceso seguro, que no pone en peligro la vida del paciente.

En la muestra, hemos incluido pacientes con antecedentes de síntomas de abstinencia graves previos, pues no lo consideramos indicación de ingreso: pensamos que dosis altas de fármacos previenen adecuadamente la sintomatología grave. Probablemente esta sólo aparece cuando, por cualquier motivo (ingreso por accidente de tráfico, por ejemplo), el paciente deja de beber repentinamente sin que se prescriban fármacos que la prevengan.

Otra indicación que no contemplamos, es la petición del paciente de ingreso. Al menos en nuestra área, no hay disponibilidad de camas hospitalarias para cumplir esto. Además, en muchos casos, lo que el paciente solicita es un internamiento más prolongado, lo que excede el supuesto a estudio.

Por todo ello, la indicación de desintoxicación ambulatoria realizada en la Unidad especializada den- 
tro del proceso asistencial, puede llevarse a cabo teniendo en cuenta las contraindicaciones señaladas. La Atención Primaria de Salud debe colaborar en el seguimiento diario del proceso.

\section{REFERENCIAS}

1. Rodríguez - Martos, A. (1995). Tratamiento del síndrome de dependencia alcohólica. Papel de la Atención Primaria, 15, 44 - 57.

2. Hayashida, M. (1989). Comparative effectiveness and costs of inpatient and outpatient detoxification of patients with mild - to - moderate alcohol withdrawal syndrome. NEJM, 320, 358 - 365.

3. Fleeman, N. (1997). Alcohol home detoxification: a literature review. Alcohol \& alcoholism, 32, 649 $-656$.

4. Holbrook, A. (1999). Meta - analysis of benzodiazepine use in the treatment of alcohol withdrawal. CMAJ, 160, $649-655$.
5. Mayo Smith, M. (1997). Pharmacological management of alcohol withdrawal. JAMA, 278, $144-151$.

6. Shaw, G. K. (1995). Detoxification: the use of Benzodiazepines. Alcohol \& alcoholism, 30, 765 - 770.

7. Morgan, M. Y. (1995). The management of alcohol withdrawal using Chlormethiazole. Alcohol \& alcoholism, 30, 771 - 774.

8. Peters, D. H. (1994). Tiapride: a review of its pharmacology and therapeutic potential in the management of alcohol dependence syndrome. Drugs, 47:1010 - 1032.

9. Williams, D. (1998). The drug treatment of alcohol withdrawal symptoms: a systematic review. Alcohol \& alcoholism, 33, $103-115$.

10. Saitz, R. (1994). Individualized treatment for alcohol withdrawal: a randomized double - blind controlled trial. JAMA, 272, 519 - 523.

11. Swift, R. (1999). Drug therapy for alcohol dependence. NEJM, 340, 1482 - 1490.

12. Garbutt, J. (1999). Pharmacological treatment of alcoholic addiction. JAMA, 28, 1318 - 1325. 